\title{
Video Article \\ Extraction of Lignin with High $\beta-0-4$ Content by Mild Ethanol Extraction and Its Effect on the Depolymerization Yield
}

\author{
Douwe S. Zijlstra ${ }^{1}$, Alessandra de Santi ${ }^{2}$, Bert Oldenburger ${ }^{1,2}$, Johannes de Vries ${ }^{2,3}$, Katalin Barta ${ }^{2}$, Peter J. Deuss ${ }^{1}$ \\ ${ }^{1}$ Department of Chemical Engineering (ENTEG), University of Groningen \\ ${ }^{2}$ Stratingh Institute for Chemistry, University of Groningen \\ ${ }^{3}$ Leibniz-Insitut f ür Katalyse e.V., Universität Rostock
}

Correspondence to: Peter J. Deuss at p.j.deuss@rug.nl

URL: https://www.jove.com/video/58575

DOI: doi: $10.3791 / 58575$

Keywords: Chemistry, Issue 143, Lignocellulose, fractionation, lignin, 2D-NMR, depolymerization, acetals, phenols

Date Published: 1/7/2019

Citation: Zijlstra, D.S., de Santi, A., Oldenburger, B., de Vries, J., Barta, K., Deuss, P.J. Extraction of Lignin with High $\beta-O-4$ Content by Mild Ethanol Extraction and Its Effect on the Depolymerization Yield. J. Vis. Exp. (143), e58575, doi:10.3791/58575 (2019).

\section{Abstract}

Lignin valorization strategies are a key factor for achieving more economically competitive biorefineries based on lignocellulosic biomass. Most of the emerging elegant procedures to obtain specific aromatic products rely on the lignin substrate having a high content of the readily cleavable $\beta-O-4$ linkage as present in the native lignin structure. This provides a miss-match with typical technical lignins that are highly degraded and therefore are low in $\beta-0-4$ linkages. Therefore, the extraction yields, and the quality of the obtained lignin are of utmost importance to access new lignin valorization pathways. In this manuscript, a simple protocol is presented to obtain lignins with high $\beta$-O-4 content by relatively mild ethanol extraction that can be applied to different lignocellulose sources. Furthermore, analysis procedures to determine the quality of the lignins are presented together with a depolymerization protocol that yields specific phenolic 2-arylmethyl-1,3-dioxolanes, which can be used to evaluate the obtained lignins. The presented results demonstrate the link between lignin quality and potential for the lignins to be depolymerized into specific monomeric aromatic chemicals. Overall, the extraction and depolymerization demonstrates a trade-off between the lignin extraction yield and the retention of the native aryl-ether structure and thus the potential of the lignin to be used as the substrate for the production of chemicals for higher-value applications.

\section{Video Link}

The video component of this article can be found at https://www.jove.com/video/58575/

\section{Introduction}

For the chemical industry to become sustainable, renewable feedstocks such as lignocellulosic biomass should be used as alternative to current dominant fossil ones ${ }^{1}$. However, to make the use of such feedstocks economically viable, high-value applications should be sought for all its contents. Lignocellulosic biomass can contain about $30 \mathrm{wt} \%$ of the lignin that is an aromatic biopolymer for which currently only a few applications have been developed beyond its use as low-value fuel ${ }^{2}$. Therefore, the methodology towards potentially increased-value aromatic components is of major interest to ensure the success of future biorefineries.

Recent research has focused heavily on the development of novel methodologies for the selective cleavage of the most abundant $\beta$-O-4 linkage (Figure 1a) in lignin to obtain specific aromatic, typically phenolic, monomers ${ }^{3,4,5,6}$. For example, the application of acids between $80^{\circ} \mathrm{C}$ to 180 ${ }^{\circ} \mathrm{C}$ is very effective in cleaving the $\beta-\mathrm{O}-4$ linkage forming aldehyde and ketone fragments ${ }^{7,8}$. Our groups and others have recently demonstrated that acidolysis combined with the methodologies to stabilize and trap reactive fragments is extremely potent to obtain phenolic monomers with specific chemical motifs ${ }^{9,10,11,12}$. Of these, in particular acetal trapping of reactive aldehydes with alcohols to obtain phenolic 2-arylmethyl-1,3dioxolanes (acetals) proved powerful due to its relative simple application and the retention of the highly functionalized nature of the lignin monomers (Figure 1b) ${ }^{13,14}$. These acetals are obtained from the depolymerization in a ratio that relates to the distribution of the $\mathrm{H}, \mathrm{G}$ and $\mathrm{S}$ monomers present in the parent lignin feedstock.

Acid catalyzed depolymerization, like many of the most elegant methodologies developed, are relatively mild and do not cleave stronger $\mathrm{C}$-C bonds that occur in lignin ${ }^{15}$. However, $\mathrm{C}-\mathrm{C}$ bonds become especially abundant when harsh lignocellulose fractionation conditions are applied due to the condensation of reactive fragments released from the cleavage of weaker $\mathrm{C}-\mathrm{O}$ bonds ${ }^{16,17}$. The loss of $\beta-\mathrm{O}-4$ content from the biomass processing method is clearly demonstrated by the analysis of a range of technical lignins, which were shown to only retain up to $6 \beta$ O-4 linkages per 100 aromatic units ${ }^{18}$, while in lignocellulose these numbers range from 45 to 90 linkages per 100 aromatic units depending on the source ${ }^{16}$. Turning to milder extraction conditions, lignins can be obtained with the linkage distributions that better reflect the natural lignin. Nevertheless, this demands a trade-off between the extraction efficiency and the quality of the obtained lignin material ${ }^{17}$. This also has precedence in organosolv extraction of lignin, which is a popular method to fractionate lignin. Many variations of this process exist, with the methods employing different temperatures, acid content, extraction times and solvents. Here, the extraction severity has a direct impact on the obtained lignin structure and thus its suitability for further valorization ${ }^{19,20,21}$. For example, organosolv lignin produced by the ethanol based Alcell process, operated for 5 years at demonstration scale, had relatively low amount of $\beta-0-4$ linkages left as it was operated at relatively high 
temperature to ensure efficient delignification in order to obtain high quality carbohydrates for bioethanol production. Nevertheless, biobased solvents with little environmental impact like ethanol are preferred and thus the extraction methods that result in lignins of higher value are of interest. Alcoholic solvents are of particular interest as in addition to being the extraction medium they also incorporate into the lignin structure, for example, $\beta^{\prime}-\mathrm{O}-4$ (Figure 1a) ${ }^{22}$, which partly "protects" the structure from undesired cleavage. A suitable method would potentially be first to obtain lignin with a high $\beta-\mathrm{O}-4$ content and in a sequential step to remove the remainder of the lignin to access high value cellulose.

In this manuscript, we describe a straight forward and highly reproducible procedure for the extraction of high $\beta-0-4$ lignin by mild ethanol extraction. Depending on the biomass source, this can lead to relatively high extraction efficiency and yield. Procedures for the characterization of the obtained lignin are provided as well as how to "deprotect" the etherified $\beta^{\prime}-0-4^{20}$. Additionally, an assessment procedure is presented for the potential of these lignins in selective depolymerization procedures that rely on selective cleavage of the $\beta-O-4$ linkages. This assessment is performed using the iron(III) triflate catalyzed depolymerization in the presence of ethylene glycol to obtain phenolic 2-arylmethyl-1,3dioxolanes ${ }^{23}$ which demonstrates the link between $\beta-O-4$ content in the lignin material and the monomer yields ${ }^{21}$. The results show the balance between high lignin extraction efficiency and the potential of the obtained lignin to be depolymerized to specific aromatic monomers.

\section{Pretreatment of the Walnut Feedstock before Lignin Extraction}

1. Production of cut walnut shells

1. Feed the walnut shells to a hammer cutter to fracture the shells. Equip the hammer cutter with a $5 \mathrm{~mm}$ sieve at the outlet. Collect the fractured walnut shells in a $1 \mathrm{~L}$ glass beaker.

2. Feed the fractured shells to a micro hammer cutter to obtain ground shells. Equip the micro hammer cutter with a $2 \mathrm{~mm}$ sieve at the outlet. Collect the ground walnut shells in a $1 \mathrm{~L}$ glass beaker.

2. Extraction of fatty acids from ground walnut shells

1. Put $150 \mathrm{~g}$ of the cut walnut shells in a $500 \mathrm{~mL}$ round-bottom flask. Add $200 \mathrm{~mL}$ of toluene and a stirring bar to the round-bottom flask.

2. Attach a reflux condenser to the round-bottom flask. Heat the mixture at reflux temperature $\left(111^{\circ} \mathrm{C}\right)$ with an oil bath for $2 \mathrm{~h}$ with vigorous stirring.

3. Stop the heating after $2 \mathrm{~h}$ and let the mixture cool down to room temperature by removing it from the oil bath.

4. Remove the toluene by filtration ( $185 \mathrm{~mm}$ diameter, $10 \mu \mathrm{m}$ pore size). Discard the toluene filtrate.

5. Remove the toluene residues by heating the walnut shells overnight in a vacuum oven at $80{ }^{\circ} \mathrm{C}$ and $50 \mathrm{mbar}$.

3. Milling of pre-extracted walnut shells

1. Place $7 \mathrm{ZrO}_{2}$ grinding balls with a $20 \mathrm{~mm}$ diameter into a $250 \mathrm{~mL}$ grinding bowl made of $\mathrm{ZrO}_{2}$.

2. Fill the bowl with $40 \mathrm{~g}$ of walnut particles. Add $60 \mathrm{~mL}$ of isopropanol to the grinding bowl.

3. Perform the grinding of the walnut shells with a rotary ball mill. Grind in 4 cycles of 2 min grinding at $27 \times g$ followed by a 4 min pause. Keep the temperature of the bowl below $80^{\circ} \mathrm{C}$ at any time. Perform no more than 3 batches and let the bowl cool down afterwards.

4. Collect the finely ground walnut shells into a $500 \mathrm{~mL}$ round-bottom flask. Remove the isopropanol by rotary evaporation at $40{ }^{\circ} \mathrm{C}$ and 125 mbar.

5. Dry the walnut shells overnight in a vacuum oven at $50{ }^{\circ} \mathrm{C}$ and $50 \mathrm{mbar}$

6. Sieve the finely ground walnut shells through a $1 \mathrm{~mm}$ sieve. Ground the particles that are too large again with the rotary ball mill.

\section{Preparation of the Wood Feedstocks}

1. Cutting of the wooden planks

1. Place the wooden planks under a drill, which is equipped with a flat wood speed drill bit. Collect the wood shavings in a glass beaker.

2. Place the wooden shavings in a coffee grinder to cut them into smaller pieces.

2. Extraction of fatty acids from wood

1. Perform the extraction of the fatty acids from wood in precisely the same manner as described for walnut shells in Step 1.2 . NOTE: No milling of the wood is performed in the ball mill, as the conditions described in Step 1.3 did not result in a reduction of the particle size.

\section{Extraction of High $\beta-0-4$ Ethanosolv Lignin}

1. Mild ethanol extraction (Method A)

1. Put $25 \mathrm{~g}$ of the feedstock in a $500 \mathrm{~mL}$ round-bottom flask. Add an $80: 20$ ethanol/water mixture $(200 \mathrm{~mL}), 4 \mathrm{~mL}$ of $37 \% \mathrm{HCl}$ solution $(0.24 \mathrm{M})$ and a magnetic stirring bar to the round-bottom flask.

2. Attach a reflux condenser to the round-bottom flask. Heat the mixture atreflux temperature with an oil bath for $5 \mathrm{~h}$ with vigorous stirring.

3. Allow the mixture to cool to room temperature by removing it from the oil bath. Filter the mixture ( $185 \mathrm{~mm}$ diameter, $10 \mu \mathrm{m}$ pore size) and wash the residue with 4 times $25 \mathrm{~mL}$ of ethanol.

2. Work-up and isolation of lignin

1. Collect the liquor in a $500 \mathrm{~mL}$ round-bottom flask. Concentrate the liquor by rotary evaporation at $40{ }^{\circ} \mathrm{C}$ and $150 \mathrm{mbar}$.

2. Dissolve the obtained solid in $30 \mathrm{~mL}$ of acetone. Use an ultrasonic bath if the solid does not dissolve completely. 
3. Precipitate the lignin by adding the mixture to $600 \mathrm{~mL}$ of water. If no precipitation occurs, add a small amount of saturated aqueous $\mathrm{Na}_{2} \mathrm{SO}_{4}$ solution to flocculate the lignin.

4. Collect the lignin by filtration ( $185 \mathrm{~mm}$ diameter, $10 \mu \mathrm{m}$ pore size). Wash the lignin with $25 \mathrm{~mL}$ of water 4 times. Discard the filtrate if no analysis of the hemicellulose fraction is required. If the filtrate is very turbid, add it to a centrifugation tube and collect the (solid) bottom fraction by centrifugation.

5. Allow the lignin to air dry overnight. Dry the lignin further in a vacuum oven (overnight at $50{ }^{\circ} \mathrm{C}$ and $50 \mathrm{mbar}$ ).

6. Determine the yield after the lignin is dried overnight in a vacuum oven.

7. Determine the lignin extraction efficiency by dividing it with the total lignin content as determined by the Klason method ${ }^{24}$.

3. Higher temperature ethanol extraction (Method B)

1. Put $15 \mathrm{~g}$ of the feedstock in a $250 \mathrm{~mL}$ autoclave. Add an $80: 20$ ethanol/water mixture $(120 \mathrm{~mL}), 2.4 \mathrm{~mL}$ of $\mathrm{HCl}(0.24 \mathrm{M})$ and a magnetic stirring bar.

2. Heat the mixture at $120^{\circ} \mathrm{C}$ for $5 \mathrm{~h}$ with a stirring speed of $5.2 \times \mathrm{g}$. Cool the mixture afterwards in an ice bath.

3. Filter the mixture ( $185 \mathrm{~mm}$ diameter, $10 \mu \mathrm{m}$ pore size) and wash the residue 4 times with $15 \mathrm{~mL}$ of ethanol.

4. Perform further work-up and isolation precisely as described in Step 3.2.

4. Larger scale higher temperature ethanol extraction of walnut shell (Method $C^{*}$ )

1. Put $90 \mathrm{~g}$ of finely ground walnut shell in a $1 \mathrm{~L}$ high pressure autoclave. Add an $80: 20$ ethanol/water mixture $(750 \mathrm{~mL})$ and $6.25 \mathrm{~mL}$ of $\mathrm{H}_{2} \mathrm{SO}_{4}(0.12 \mathrm{M})$.

2. Heat the mixture at $120{ }^{\circ} \mathrm{C}$ for $5 \mathrm{~h}$ with a stirring speed of $35.8 \times \mathrm{g}$. Cool the mixture back to room temperature by turning on the reactors cooling system after the $5 \mathrm{~h}$ reaction time.

3. Filter the mixture ( $185 \mathrm{~mm}$ diameter, $10 \mu \mathrm{m}$ pore size) and wash the residue 4 times with $75 \mathrm{~mL}$ of ethanol. NOTE: Using multiple filters saves a lot of time.

4. Collect the liquors in 2 equal batches. Perform the work-up and isolation as described as in Step 3.2 with double amount of the solvents for both batches.

5. Control experiments of Step 3.1. (Method $A^{*}$ ) and Step 3.3. (Method $\mathbf{B}^{*}$ ) (optional)

1. Put the exact some materials as described in Step 3.1.1 but replace the $\mathrm{HCl}$ solution with $1.67 \mathrm{~mL}$ of $\mathrm{H}_{2} \mathrm{SO}_{4}(0.12 \mathrm{M})$. The rest of Step 3.1-3.2 is identical to Method A.

2. Put the exact some materials as described in Step 3.3.1 but replace the $\mathrm{HCl}$ solution with $1.0 \mathrm{~mL}$ of $\mathrm{H}_{2} \mathrm{SO}_{4}(0.12 \mathrm{M})$. The rest of Step 3.3 is identical to Method B.

\section{De-etherification of the Lignin (optional)}

1. Dissolve $1000 \mathrm{mg}$ of lignin in a $24 \mathrm{~mL}$ of $1: 1$ 1,4-dioxane/water mixture in a $100 \mathrm{~mL}$ round-bottom flask. Add $1 \mathrm{~mL}$ of a $37 \% \mathrm{HCl}$ solution to the mixture.

2. Add a stirring bar and attach a reflux condenser to the round-bottom flask. Heat the mixture to $100{ }^{\circ} \mathrm{C}$ with an oil bath for $5 \mathrm{~h}$ with vigorous stirring.

3. Allow the mixture to cool down to room temperature by removing it from the oil bath. Add the mixture to $160 \mathrm{~mL}$ of water to precipitate the lignin.

4. Collect the lignin by filtration ( $185 \mathrm{~mm}$ diameter, $10 \mu \mathrm{m}$ pore size) and wash the lignin with $25 \mathrm{~mL}$ of water 2 times. Allow the lignin to air dry overnight. Dry the lignin further in a vacuum oven (overnight at $50{ }^{\circ} \mathrm{C}$ and $50 \mathrm{mbar}$ ).

\section{Analysis of Lignin}

1. Two-dimensional nuclear magnetic resonance (2D-NMR) analysis

1. Dissolve $60 \mathrm{mg}$ of dried lignin in $0.7 \mathrm{~mL}$ of $d_{6}$-acetone. Add a few drops of $D_{2} \mathrm{O}$ if the lignin does not fully dissolve. Put the mixture in an NMR-tube and take a 2D proton heteronuclear single quantum coherence spectra (HSQC) with an NMR spectrometer with the following parameters: $(11,-1),(160,-10), n t=4, n i=512^{20}$.

2. Analyze the obtained HSQC spectra. Adjust the spectra by manual phase corrections on both axis until all signals are positive, as this is especially important along the horizontal (f2) axis. Perform no baseline corrections. The positions of all the linkages are given in Step 5.1 .3 and 5.1.6

3. Integrate the signals in the aromatic region that correspond to the three different aromatic units (proton numbering as per Figure 4). These signals are in the region [(Proton range)(Carbon range)]:
$\mathrm{S}_{2 / 6}: \quad[(6.48-6.90)(104-109)]$
$\mathrm{S}_{2 / 6}^{\prime}: \quad[(7.17-7.50)(105-109)]$
$S_{\text {condensed: }} \quad[(6.35-6.65)(106-109)]$
$\mathrm{G}_{2}: \quad[(6.78-7.14)(111.5-116)]$
$\mathrm{G}_{5}: \quad[(6.48-7.06)(115-120.5)]$
$\mathrm{G}_{6}: \quad[(6.65-6.96)(120.5-124.5)]$
$\mathrm{H}_{2 / 6}: \quad[(7.05-7.29)(128.5-133)]$

Note: $H_{3 / 5}$ overlaps with the $G_{5}$ signal, and it is assumed that $H_{2 / 6}$ has the same intensity as $H_{3 / 5}$. The signal for condensed $G$ overlaps with $G_{5}$. If no (or hardly any) $G_{2}$ and $G_{6}$ signals are present, this indicates that full condensation of $G$ has occurred.

4. Calculate the amount of aromatic units with the formula:

Total aromatic $=\left(\left(\left(S_{2 / 6}+S_{2 / 6}^{\prime}\right) / 2\right)+S_{\text {condensed }}\right)+\left(\left(G_{2}+G_{5}+G_{6}-H_{2 / 6}\right) / 3\right)+\left(H_{2 / 6} / 2\right)$

5. Calculate the percentage of $\mathrm{G}, \mathrm{H}$ and $\mathrm{S}$ units with the following formulas:

Ratio $S=\left(\left(\left(S_{2 / 6}+S_{2 / 6}^{\prime}\right) / 2\right)+\right.$ Scondensed $)$ : total aromatic $\times 100 \%$

Ratio $G=\left(\left(G_{2}+G_{5}+G_{6}-H_{266}\right) / 3\right)$ : total aromatic $\times 100 \%$ 
Ratio $\mathrm{H}=\left(\mathrm{H}_{2 / 6} / 2\right)$ : total aromatic $\times 100 \%$

6. Integrate the signals in the aliphatic region signals that correspond to the $\beta-O-4, \beta-\beta$ and $\beta-5$ linkages and Hibbert Ketones. These are in the region [(proton range)(carbon range)]:

$\beta-O-4_{\alpha} \quad[(4.76-5.10)(73-77.5)]$

$\beta^{\prime}-0-4_{\alpha} \quad$ [(4.44-4.84)(81.5-86)]

$\beta-O-4_{\beta}$ and $\beta^{\prime}-0-4_{\beta} \quad[(4.03-4.48)(85-90.5)]$

$\beta-O-4_{\gamma}$ and $\beta^{\prime}-O-4_{\gamma} \quad[(3.10-4.00)(58.5-62)]$

$\beta-5_{\alpha} \quad$ [(5.42-5.63)(88-92)]

$\beta-5_{\beta} \quad[(3.36-3.56)(53-54.5)]$

$\beta-5_{\gamma} \quad[(3.50-4.00)(62-64.5)]$

$\beta-\beta_{\alpha} \quad[(4.59-4.77)(86.5-89.5)]$

$\beta-\beta_{\beta} \quad[(2.98-3.20)(55.5-59)]$

$\beta-\beta_{Y} \quad[(3.75-3.96)(72.5-76)]$ and $[(4.10-4.31)(72.5-76)]$

$\mathrm{HK}_{\mathrm{y}} \quad[(4.20-4.30)(66-68)]$

Note: The $\beta$-protons of the $\beta-\mathrm{O}-4$ and $\beta^{\prime}-\mathrm{O}-4$ linkages overlap. The structural motifs of these linkages are given in Figure 1.

7. The total number of linkages per $100 \mathrm{C} 9$ units are all based on the signal of the $\alpha$ proton of the linkages. Calculate the total number of linkages with the following formulas:

$\beta-O-4$ linkages $=\left(\beta-O-4_{\alpha}+\beta^{\prime}-O-4_{\alpha}\right) /$ total aromatic $\times 100$

$\# \beta-5$ linkages $=\beta-5_{\alpha} /$ total aromatic $\times 100$

$\beta-\beta_{\text {linkages }}=\beta-\beta_{\alpha} /$ total aromatic $\times 100$

2. Gel permeation chromatography (GPC) analysis

1. Dissolve $10 \mathrm{mg}$ of dried lignin in $1 \mathrm{~mL}$ of tetrahydrofuran (THF) (with a drop of toluene as the internal standard). Filter this mixture through a $0.45 \mu \mathrm{m}$ syringe filter into an autosampler vial with a reduced volume inlet of $0.3 \mathrm{~mL}$. Close the autosampler vial with a cap.

2. Inject $20 \mu \mathrm{L}$ of the sample into a THF GPC. Process the obtained data.

3. Correct the obtained signal for the reference signal (toluene). Select the elution volume for the correct range ( 200-10000 Da). Calculate mass distribution by the software.

\section{Depolymerization of Lignins to Phenolic 2-Arylmethyl-1,3-Dioxolanes (Acetals)}

1. Place $50 \mathrm{mg}$ of dried lignin in a $20 \mathrm{~mL}$ microwave vial as the reaction vessel equipped with a magnetic stirrer. Add $0.85 \mathrm{~mL}$ of 1,4 -dioxane, 50 $\mu \mathrm{L}$ of ethylene glycol in 1,4-dioxane $(0.54 \mathrm{~mL} / \mathrm{mL})$ and $50 \mu \mathrm{L}$ of octadecane (internal standard) in 1,4-dioxane $(26 \mathrm{mg} / \mathrm{mL})$.

2. Close the reaction vessel and heat the solution to $140{ }^{\circ} \mathrm{C}$ while stirring at $3.8 \times \mathrm{g}$.

3. When the reaction vessel reaches $140{ }^{\circ} \mathrm{C}$, add $50 \mu \mathrm{L}$ of $\mathrm{Fe}(\mathrm{III}) \mathrm{OTf}{ }_{3}$ in 1 ,4-dioxane $(0.1 \mathrm{~g} / \mathrm{mL})$.

4. Stir the reactor for $15 \mathrm{~min}$.

5. Cool the reactor to room temperature and remove the depolymerization liquid as described in Step 7.1.

\section{Work up and Analysis of Depolymerization Mixtures}

1. Filter the liquid over Celite (permeability: 2.60-6-50 darcy; particle size: 150 mesh Tyler; sieve retain (140 M US): $2.0-25.0 \%$ ) and collect in a $2 \mathrm{~mL}$ centrifuge tube.

2. Concentrate the liquid overnight at $35^{\circ} \mathrm{C}$ in a rotational vacuum concentrator.

3. Extract the final oil/solid with the following procedure:

1. Suspended and swell the residue in $0.15 \mathrm{~mL}$ of dichloromethane (DCM) by extensive mixing (by vortex), 15 min of sonication and 30 min in automatic wheel.

2. Centrifuge the samples for up to $10 \mathrm{~s}$ using a minispin tabletop centrifuge to ensure that the liquid is at the bottom of the tube (centrifugation speed: $671 \times \mathrm{g}$ ).

3. Add $0.75 \mathrm{~mL}$ of toluene and mix extensively (by vortex and $10 \mathrm{~min}$ sonication).

4. Centrifuge the samples for up to $10 \mathrm{~s}$ using a minispin tabletop centrifuge (centrifugation speed: $671 \times \mathrm{g}$ ) to ensure that the liquid is at the bottom of the tube.

5. Separate the light organic liquid from the solid or thick oily residue and filter this liquid over a plug of Celite and collect in a glass vial. NOTE: This procedure for suspension/washing is repeated three times, and in the last extraction, $0.5 \mathrm{~mL}$ of toluene is used.

4. Concentrate the combined organic phases by rotary evaporation $\left(40{ }^{\circ} \mathrm{C}, 20 \mathrm{mbar}\right)$.

5. Dissolve the oily residue in $1 \mathrm{~mL}$ of DCM for gas chromatography flame ionization detector (GC-FID) analysis.

6. Perform GC-FID using a GC equipped with an FID detector and use helium as the carrier gas. Standard settings: $1 \mu \mathrm{L}$ injection, a split ration of $50: 1$, a helium flow of $0.95 \mathrm{~mL} / \mathrm{min}$. Equip the GC apparatus with a HP5 column $(30 \mathrm{~m} \times 0.25 \mathrm{~mm} \times 0.25 \mu \mathrm{m})$ and run with a temperature profile which starts with a $5 \mathrm{~min} 60^{\circ} \mathrm{C}$ isotherm. Follow-up by a $10^{\circ} \mathrm{C} / \mathrm{min}$ ramp for $20 \mathrm{~min}$ to $260^{\circ} \mathrm{C}$. Hold this temperature for 20 min.

7. Integrate the peaks in the spectra manually. The retention times of the peaks are as following: octadecane (21.4 min), $\mathrm{H}$-acetal (19.5 min), Gacetal $(20.8 \mathrm{~min}$ ), S-acetal $(23.4 \mathrm{~min})$. Use the obtained values in Step 7.8 to perform the quantification.

8. Perform the quantification of $\mathrm{G}$ acetal, which is based on a calibration curve with a standard compound isolated using an internal standard (octadecane).

NOTE: Calibration curve: $\frac{\text { grams } G \text { acetal }}{\text { grams octadecane }}=1,94 * \frac{\text { Area } G \text { acetal }}{\text { Area octadecane }}+0,042\left(R^{2}=0,9991\right)$

Yield G-acetal: $\frac{\text { grams G acetal }}{\text { grams lignin }} * 100$

Based on previous results ${ }^{9,21,23}$ a response factor was estimated for $\mathrm{H}$ and $\mathrm{S}$ acetal as 2.19 and 1.82, respectively. 
Representative Results

In Figure 2, the obtained feedstock after pretreatment are shown (left column). All feedstock was obtained as small chips present apart from beech wood, which was acquired as shavings of suitable particle size for extraction. The lignins obtained after the extraction show a wide range of colors and particle sizes. The lignins obtained from mild treatments (method A and second column Figure 2) are typically red/pink in color and acquired as small flakes. When harsher conditions are applied (methods $B$ and $C^{*}$ ), the obtained lignins have a brown/brownish yellow color (third and fourth column Figure 2). The yield did increase for all the extractions performed under harsher conditions (methods B and $\mathrm{C}^{*}$ ) compared to milder conditions (Reaction scheme in Figure 1, results in Table 1). This effect was much more profound for walnut (10.2\% increase), beech (8.5\% increase) and cedar wood (5.1\% increase) compared to pine wood (only $0.5 \%$ increase). Based on the lignin content of the biomass before extraction ( $40.3 \%$ for walnut, $28.6 \%$ for pine ${ }^{25}, 18.8 \%$ for beech ${ }^{25}$ and $35.1 \%$ for cedar ${ }^{25}$ ), the lignin extraction efficiency of beech wood is especially high $(73.9 \%)$, whereas for the other sources lower extraction efficiency was obtained. Methods $A^{*}$ and $B^{*}$, control experiments with sulfuric acid for methods $A$ and $B$, showed some clear differences in extraction yield. Mild extraction of walnut shells with sulfuric acid (method $A^{*}$ ) gave only a very low yield of $2.6 \%$, which is markedly lower than the extraction with hydrochloric acid (method $A$ ) $(2.6 \%$ and $5.0 \%)$. However, with harsher extraction conditions, the extraction with sulfuric acid (method $\mathrm{B}^{*}$ ) shows a higher yield compared to hydrochloric acid (method B) $(19.3 \%$ and $15.2 \%)$, but it should be noted that sugar traces are present in the product obtained by extraction with sulfuric acid.

From the NMR analysis of the different lignins (example shown in Figure 4), the H/G/S ratio and amount of linkages were determined (Table 1). Due to the overlap of the $\beta$ and $\gamma$-protons of the $\beta-O-4$ and the $\beta^{\prime}-O-4$ linkage, the amount of linkages is quantified using the $\alpha-p r o t o n s$. Additionally, the $\mathrm{G}_{5 / 6}$ and $\mathrm{H}_{3 / 5}$ signals overlap but these can be corrected by adjusting the ratios accordingly using the $\mathrm{H}_{2 / 6}$ signals. Also, a signal corresponding to the $\mathrm{Y}$-protons of the Hibbert Ketones and a signal for oxidized $\mathrm{S}$ units, which likely are caused by lignin end-groups, are identified.

The ratios obtained from NMR show that in general extractions with method B provides lignin with higher $S$ content compared to those with method $A$ in the case that the native material contains $S$ units. Also, the extractions with method $B$ provide lignin with a lower amount of total $\beta-O-4$ linkages compared to method $A$, indicating increased degradation upon increase in temperature. An exception is the walnut lignin obtained from methods methods $A$ and $B$ for which the amount of total $\beta-O-4$ linkages was very similar. The number of $\beta-\beta$ and $\beta-5$ linkages does decrease when harsher conditions are applied, although to a lesser extent. Additionally, NMR revealed that all the lignins obtained after ethanol extraction showed a degree of structural modification of the $\beta-\mathrm{O}-4$ linkage. These have at least $\sim 50 \%$ substitution at the $\alpha-\mathrm{OH}$ group, resulting in the $\alpha$-ethoxylated $\beta^{\prime}-\mathrm{O}-4$ linkage. The lignin extraction shows high reproducibility, which was proven by performing the mild extraction of walnut shells (method A) 4 times. Especially, the deviation in the total number of $\beta-O-4$ linkages is remarkably small. When the extraction was performed under harsher conditions (methods B and $C^{*}$ ), the percentage of $\alpha$-ethoxylation increased. In the HSQC spectra of beech lignin extracted at harsher condition (method $\mathrm{B}$ ), a signal for $\mathrm{S}$ condensed is visible, which fits perfectly with the significant decrease in the amount of $\beta-O-4$ linkages. The walnut extraction performed at large scale (method $C^{*}$ ) shows a significant decrease for all linkages and a signal for S condensed is visible in the HSQC spectra. The relatively high yield for the extraction of cedar at mild conditions (method A) is caused by the presence of a substantial amount of fatty acid. Control experiments with sulfuric acid gave good insight in the effect of the acid on the composition of the obtained lignin. With mild extraction conditions (methodA*), a very pure lignin was obtained which was similar in composition compared to the other mild extractions (methodA). The somewhat lower amount of $\beta-O-4$ linkages can be attributed to a less efficient incorporation of ethanol into the lignin framework, resulting in a lower number of $\beta^{\prime}-\mathrm{O}-4$ linkages. At harsher extraction conditions $\left(\right.$ method $\mathrm{B}^{*}$ ), the differences with the obtained lignin is much more profound compared with the lignin obtained from walnut shells extracted in the presence of hydrochloric acid (method B). The total number of $\beta-0-4$ linkages shows a sharp decrease (35 and 74 , respectively) and the lignin obtained with sulfuric acid shows a high amount of condensation in the aromatic region (48\%), which was determined by the integration of the signals corresponding the $S_{\text {condensed }}$ and $G_{\text {condensed }}$ (Step 5.1.3). This high amount of condensation can be fully attributed to sulfuric acid, as the product obtained from the same extraction with hydrochloric acid showed no condensation in the aromatic region. The composition of the product obtained at harsh larger scale extraction (method $\mathrm{C}^{*}$ ) shows no big difference with the product obtained at a smaller scale $\left(\right.$ method $\mathrm{B}^{*}$ ). The only big difference is the lower amount of condensation in the aromatic region in the large-scale extraction ( $9 \%$ ) and subsequently a higher amount of $\beta-O-4$ linkages. This difference could be caused by the difference in the heating profile between the different autoclaves.

The lignins were also analyzed by GPC (Figure 5) to provide insight in the molecular weight (Table 2). These reveal that when harsher extraction conditions (method $B$ ) are applied, both the weight average molecular weight $\left(\mathrm{M}_{\mathrm{w}}\right.$ ) and the polydispersity are increasing for all sources. The number average molecular weight $\left(\mathrm{M}_{n}\right)$ between the extraction conditions are comparable for each source. Overall, these results show that harsher extraction conditions have a two-fold effect, and larger fragments are extracted in addition to additional breakdown of such fragments.

For some applications, the formation of $\beta^{\prime}-\mathrm{O}-4$ linkage is undesired, for example, when applying depolymerization methods that rely on the oxidation of the benzylic $(\alpha)$ hydroxyl group ${ }^{26,27,28}$. The transformation of $\beta^{\prime}-\mathrm{O}-4$ linkage of ethanosolv lignin to regular $\beta-O-4$ linkages was previously reported ${ }^{20}$ and was performed with a lignin batch obtained from walnut shells that is comparable to the lignin obtained from walnut shells reported in this paper (Figure 6). This lignin consisted of 30 native $\beta-O-4$ linkages and 39 -ethoxylated $\beta^{\prime}-0-4$ linkages (34 and 38 linkages, respectively for the lignin in this paper). De-etherification converted almost all the $\alpha$-ethoxylated linkages to the native structure as the obtained lignin consisted of $57 \beta-O-4$ linkages and only $3 \alpha$-ethoxylated $\beta^{\prime}-\mathrm{O}-4$ linkages, showing a small loss in the total number of $\beta-0-4$ units. The mass of the lignin was $72 \%$ of the original lignin, which is primarily caused by the loss of the ethyl group.

To demonstrate the potential of the lignin for the production of aromatic monomers through mild depolymerization, acidolysis reations with $\mathrm{Fe}(\mathrm{OTf})_{3}$ in the presence of ethylene glycol were performed (Figure 7). This reaction yields three different phenolic 2-arylmethyl-1,3-dioxolanes (acetals) that relate to the $\mathrm{H}, \mathrm{G}$ and $\mathrm{S}$ units present in the lignin. Table 3 shows the yield of the $\mathrm{S}, \mathrm{G}$ and $\mathrm{H}$ acetals and the total yields are shown in Figure 8. It is visible that the lignin extraction method has a major effect being the yield of acetals. Lower yields are obtained for lignin extracted using harsher conditions (method B). This is likely due to a more modified (higher percentage of $\alpha$-ethoxylation) condensed structure as described in the previous paragraph. 
The importance of the $\beta-\mathrm{O}-4$ units is reflected by providing correlations to monomer yield in depolymerization such as presented in the protocol (Figure 9). A clear trend is visible considering the total $\beta-0-4$ content and the non-etherified $\beta-O-4$ linkages, where a higher $\beta-0-4$ content generally results in higher yield of phenolic 2-arylmethyl-1,3-dioxolanes (acetals) which is in line with previous results ${ }^{21}$. When considering the etherified $\beta^{\prime}-\mathrm{O}-4$ linkages, the trend is also clear, showing that the depolymerization yield is not related to the number of $\beta^{\prime}-\mathrm{O}-4$ linkages. Under reaction conditions, the etherified $\beta-\mathrm{O}-4$ linkages can be de-etherified but this additional step results in the loss of material, as described earlier

Overall, correcting the monomer depolymerization yield for the lignin extraction yield, the following results can be obtained (Table 4). These show that comparing methods A \& B, generally higher amounts of acetal can be obtained by harsher extraction providing higher overall lignin yields followed by a (less selective) depolymerization. Nevertheless, the results for pinewood also show that this is dependent on the biomass source since the increase in extraction severity does not provide a significant yield increase. Retention of the $\beta-O-4$ structure is preferred for this wood type to give higher overall phenolic 2-phenylmethyl-1,3-dioxolane (acetals) yields.

a<smiles>[R]c1cc(C(C)C)cc([R])c1OC(CO)C(O)c1cc([R])c(OCC)c(OC)c1</smiles>
$\mathrm{R}^{2}$

$$
\begin{aligned}
& \mathrm{H}: \mathrm{R}^{1}=\mathrm{R}^{2}=\mathrm{H} \quad \beta^{\prime}-\mathrm{O}-4: \mathrm{R}^{3}=\mathrm{Et} \\
& \mathrm{G}: \mathrm{R}^{1}=\mathrm{OMe}, \mathrm{R}^{2}=\mathrm{H} \\
& \mathrm{S}: \mathrm{R}^{1}=\mathrm{R}^{2}=\mathrm{OMe}
\end{aligned}
$$$$
\text { b }
$$<smiles>COc1cc(CC2OCCO2)ccc1O</smiles>

Figure 1. Chemical structures of the obtained products. (a) Common structural motifs as present in the lignin structure. (b) Acid catalyzed lignin depolymerization combined with acetal trapping to obtain phenolic 2-arylmethyl-1,3-dioxolanes (acetals). Please click here to view a larger version of this figure.

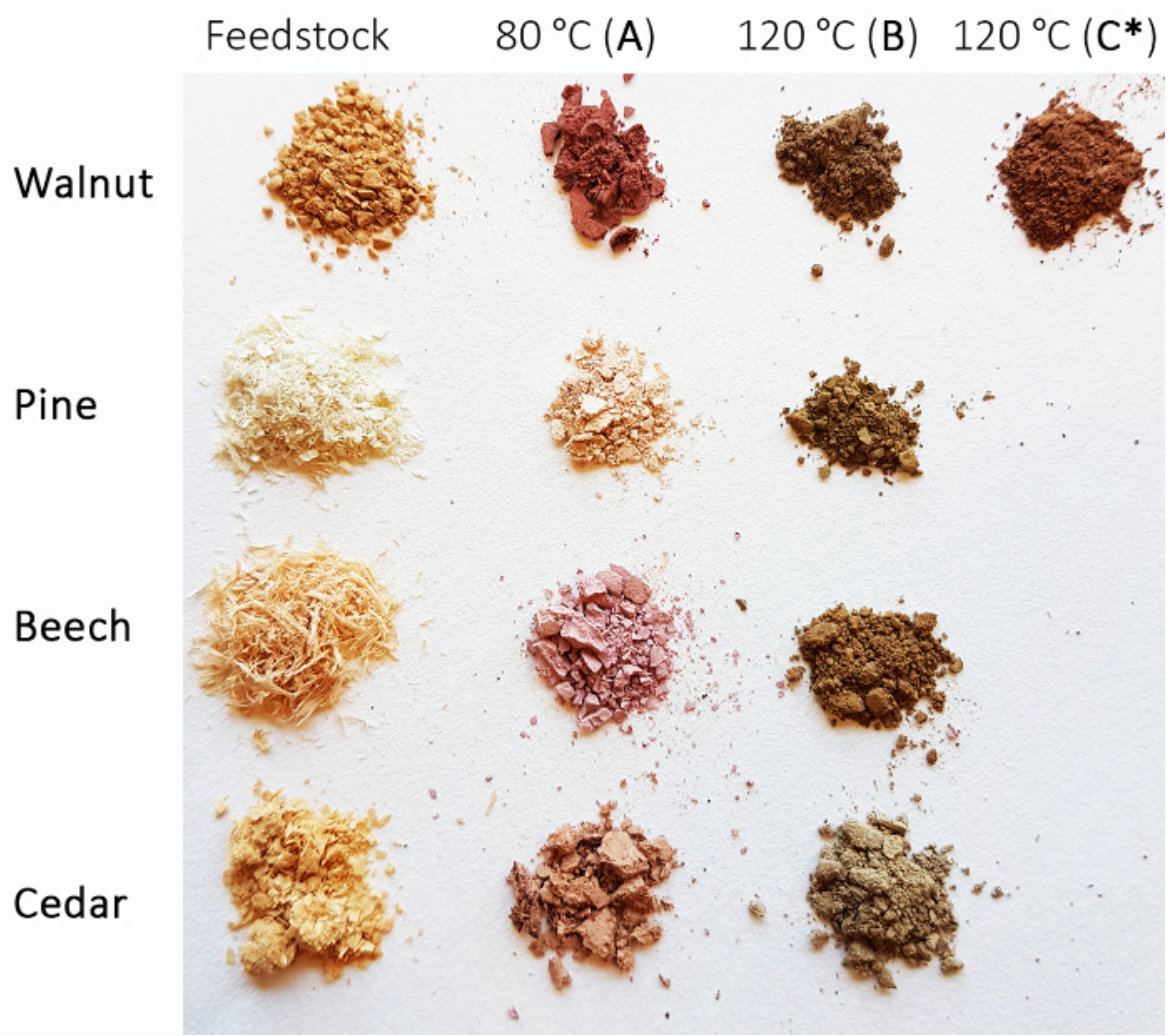

Figure 2. Obtained lignin from different feedstocks. Image of the four different lignocellulose feedstocks after pretreatment (Steps 1 and 2) and the obtained lignins after organosolv extraction at different conditions (method A-step 3.1, method B-step 3.3 and method C*-step 3.4). Please click here to view a larger version of this figure. 


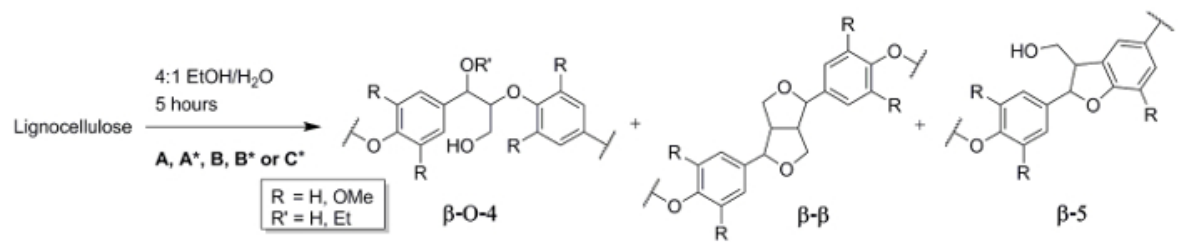

Figure 3. Reaction scheme for ethanosolv extraction. Overview of the obtained linkages: $\beta-O-4\left(R^{\prime}=H\right), \beta^{\prime}-O-4\left(R^{\prime}=E t\right), \beta-\beta$ and $\beta-5$.

Conditions: (A) $80{ }^{\circ} \mathrm{C}, 0.24 \mathrm{M} \mathrm{HCl}$ (step 3.1), (B) $120^{\circ} \mathrm{C}, 0.24 \mathrm{M} \mathrm{HCl}$ (step 3.3), (C*) $120^{\circ} \mathrm{C}, 0.12 \mathrm{M} \mathrm{H}_{2} \mathrm{SO}_{4}$ (step 3.4) and the control experiment $A^{*}$ and $B^{*}$ (step 3.5). Please click here to view a larger version of this figure.

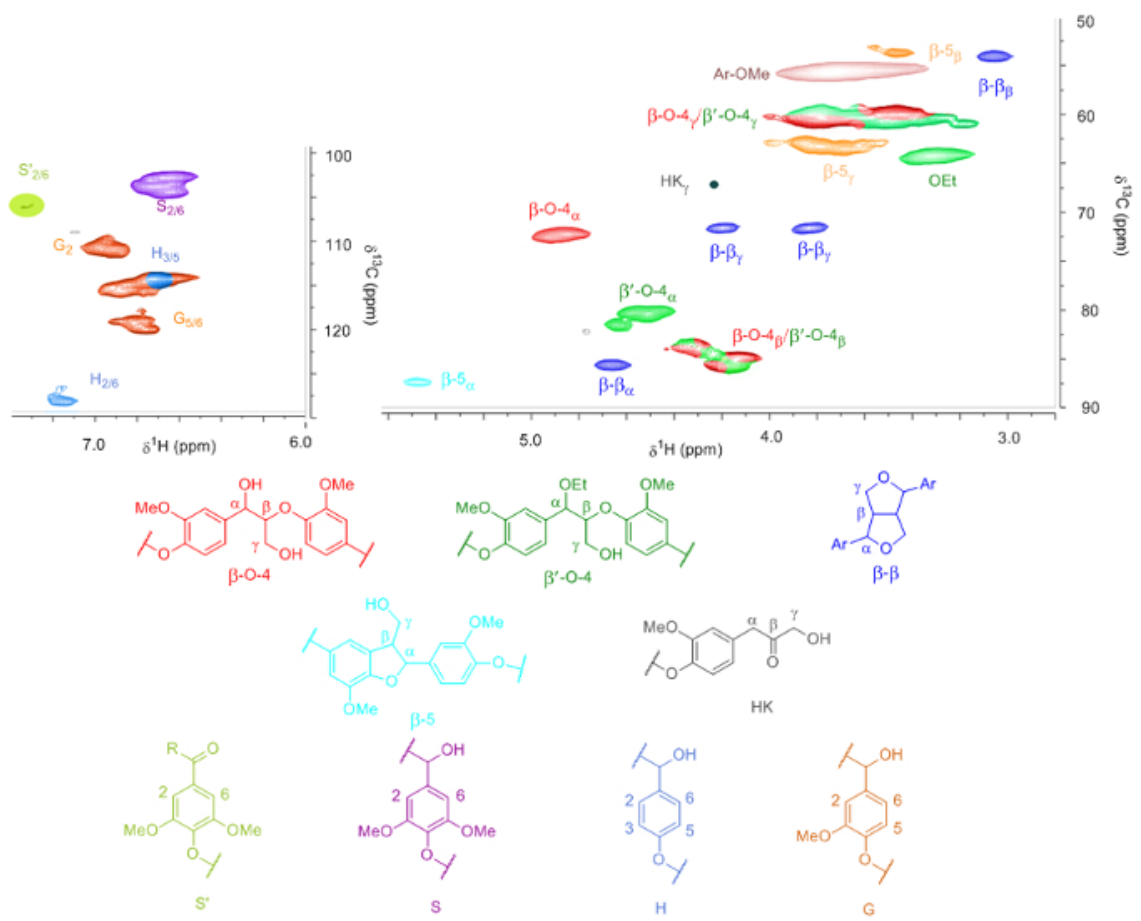

Figure 4. HSQC analysis of lignin. Identification of all lignin linkages measured with 2D-HSQC of lignin obtained from walnut shells using mild treatment (step 3.1). The signals for $\mathrm{HK}_{\mathrm{y}}$ and $\mathrm{S}_{2 / 6}^{\prime}$ are magnified to make them visible. Please click here to view a larger version of this figure.
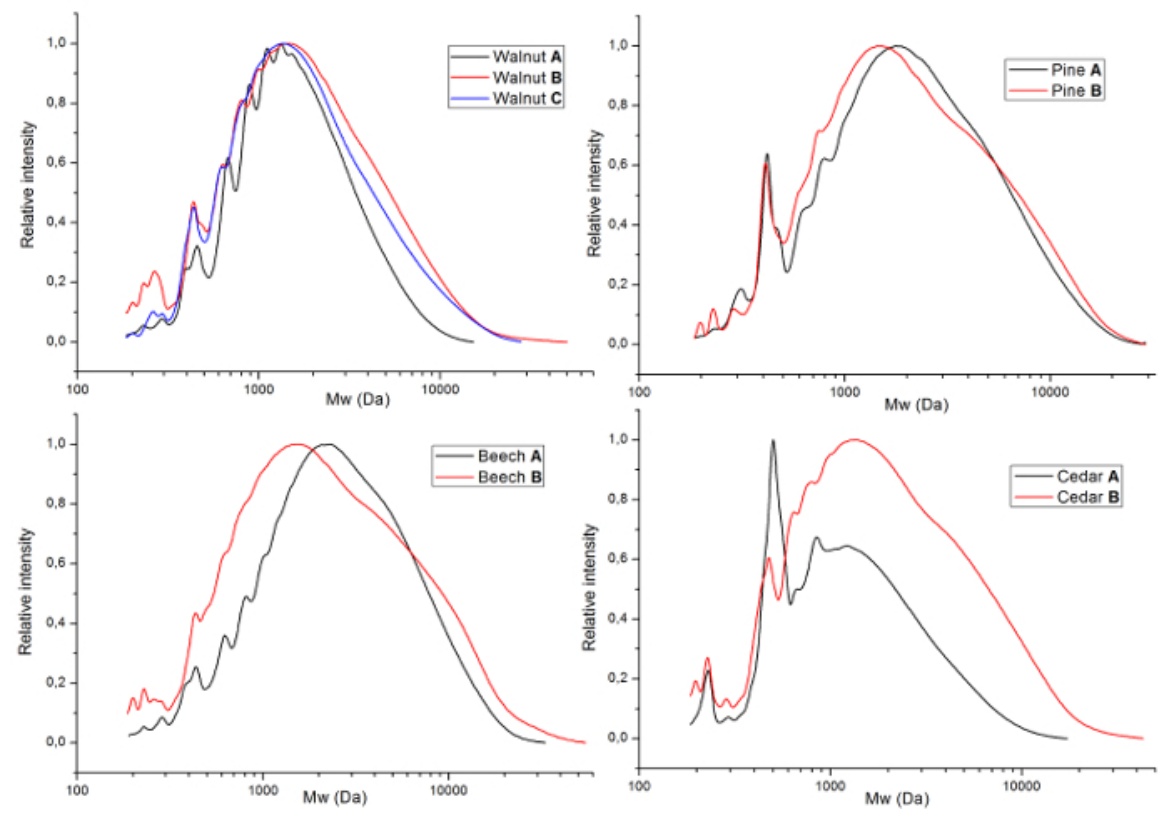

Figure 5. Molecular weight of lignin. GPC graphs of the obtained lignins divided by source $(\mathbf{a}=$ walnut, $\mathbf{b}=$ pine wood, $\mathbf{c}=$ beech wood and $\mathbf{d}$ = cedar wood). The lines correspond to different samples as given by Table 2. Please click here to view a larger version of this figure. 
<smiles>[R]c1cc(C(C)C)cc(OC)c1OC(CO)C(CC)OCCO</smiles>
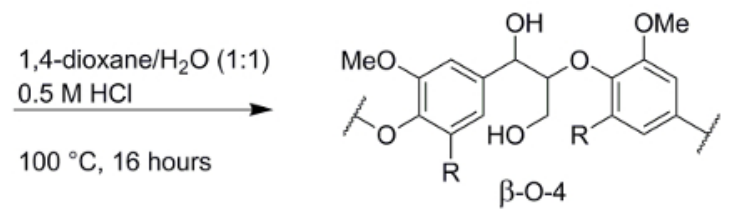

Figure 6. De-etherification of lignin. Reaction scheme of the de-etherification of the obtained ethanosolv lignin from walnut shells (step 4). Please click here to view a larger version of this figure.

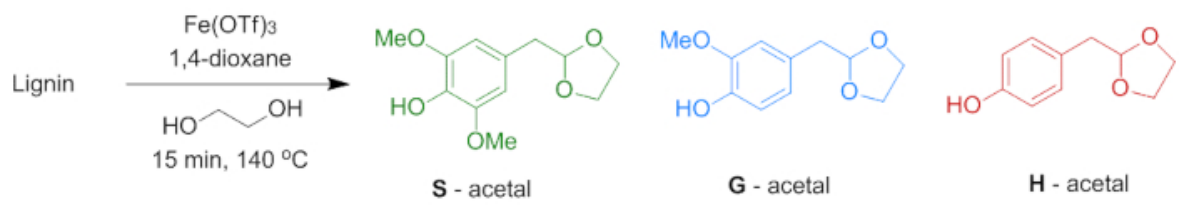

Figure 7. Lignin depolymerization to acetals. Reaction scheme for depolymerization of lignin to phenolic 2-arylmethyl-1,3-dioxolanes (acetals). $H$ unit: $R^{1}=R^{2}=H ; G$ unit: $R^{1}=O M e, R^{2}=H ; S$ unit: $R^{1}=R^{2}=O M e$ (step 6). Please click here to view a larger version of this figure.

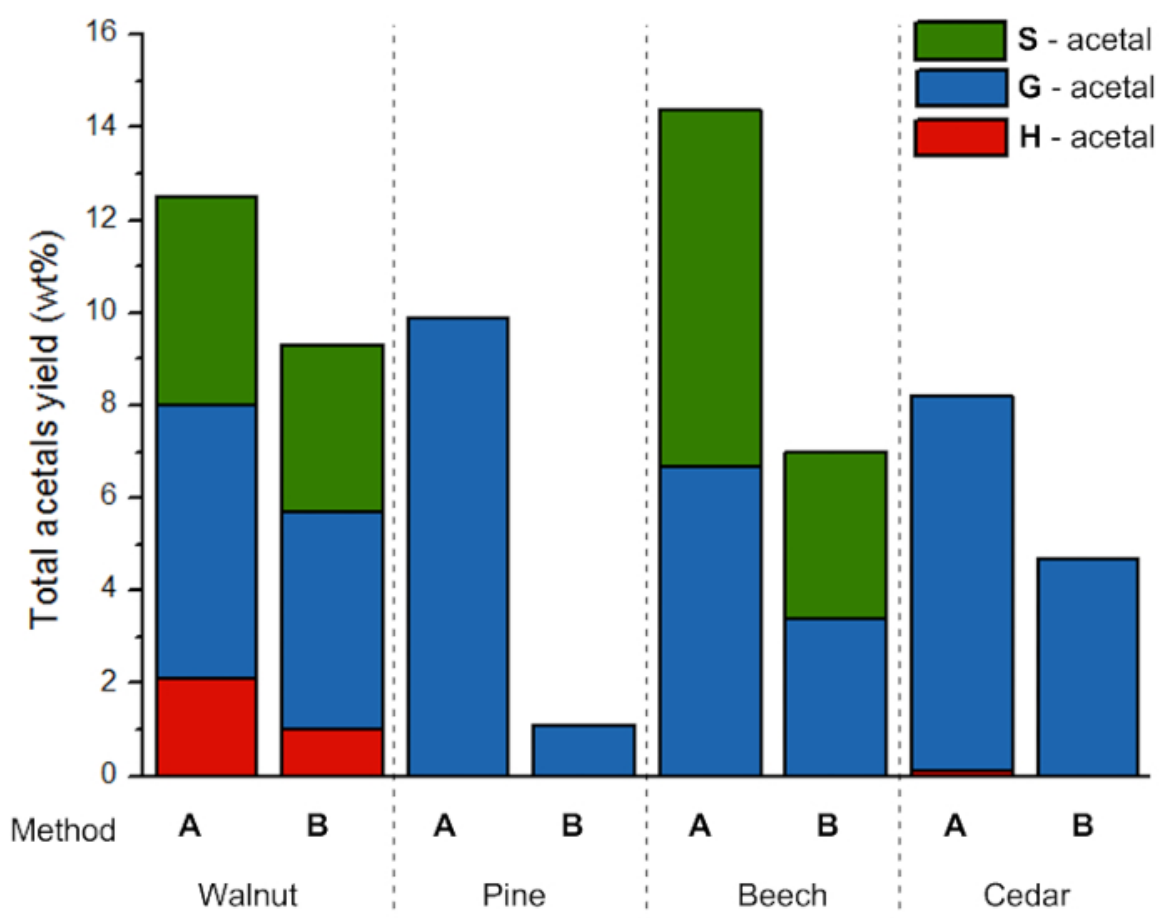

Figure 8. Acetal yield per source. Yields of phenolic 2-arylmethyl-1,3-dioxolanes (acetals) obtained from depolymerization of lignin from different sources. Please click here to view a larger version of this figure. 


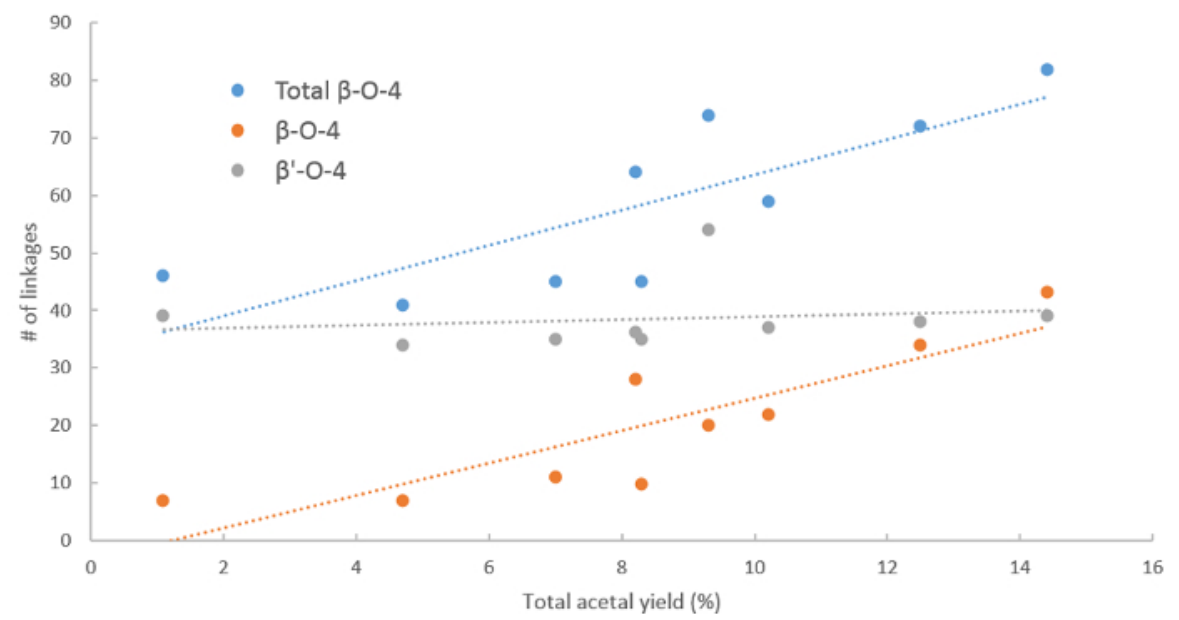

Figure 9. Influence of $\beta-0-4$ linkages on the acetal yield. Yields of phenolic 2-arylmethyl-1,3-dioxolanes (acetals) obtained from lignin depolymerization compared to the total $\beta-\mathrm{O}-4$ (blue), non-etherified $\beta-\mathrm{O}-4$ (orange) and etherified $\beta-0-4$ (gray) content in the lignin feedstock. Please click here to view a larger version of this figure.

\begin{tabular}{|c|c|c|c|c|c|c|c|c|c|}
\hline Source & Conditions & Yield (\%) & $\begin{array}{l}\text { Extraction } \\
\text { efficiency } \\
(\%)^{1}\end{array}$ & $\mathrm{~S} / \mathrm{G} / \mathrm{H}$ ratio & Total $\quad \beta-O-4$ & $\beta-O-4$ & $\beta^{\prime}-\mathrm{O}-4$ & $\beta-\beta$ & $\beta-5$ \\
\hline Walnut & $A$ & $5.0 \pm 0.7$ & 12.4 & $45 / 46 / 9$ & $75 \pm 2.5$ & $36 \pm 2.6$ & $39 \pm 3.1$ & $11 \pm 0.7$ & $5 \pm 1.5$ \\
\hline Walnut & $A^{*}$ & 2.6 & 6.5 & $47 / 45 / 8$ & 53 & 32 & 21 & 9 & 4 \\
\hline Walnut & $B$ & 15.2 & 37.7 & $59 / 37 / 4$ & 74 & 20 & 54 & 9 & 6 \\
\hline Walnut ${ }^{2}$ & $B^{*}$ & 19.3 & 47.9 & $75 / 25 / 0$ & 35 & 5 & 30 & 7 & 3 \\
\hline Walnut ${ }^{2}$ & $C^{*}$ & 16.2 & 40.2 & $65 / 33 / 2$ & 45 & 10 & 35 & 8 & 3 \\
\hline Pine & $A$ & 3.5 & 12.2 & $0 />99 /<1$ & 59 & 22 & 37 & 0 & 14 \\
\hline Pine & B & 4.0 & 14.0 & $0 />99 /<1$ & 46 & 7 & 39 & 0 & 8 \\
\hline Beech & $A$ & 5.4 & 28.7 & $63 / 37 / 0$ & 82 & 43 & 39 & 12 & 5 \\
\hline Beech $^{3}$ & B & 13.9 & 73.9 & $83 / 17 / 0$ & 45 & 11 & 35 & 9 & 2 \\
\hline Cedar & $A$ & 6.4 & 18.2 & $0 />99 /<1$ & 64 & 28 & 36 & 0 & 6 \\
\hline Cedar & $B$ & 11.5 & 32.8 & $0 />99 /<1$ & 41 & 7 & 34 & 0 & 7 \\
\hline
\end{tabular}

Table 1. Ethanosolv extraction results. Obtained yields, aromatic distribution and linkages for the different extractions performed on biomass ${ }^{*}$ Sulfuric acid is used as acid. ${ }^{1}$ Yield of lignin (wt\%)/Lignin content in the feedstock as determined by Klason lignin determination. ${ }^{2}$ Hemicellulose and S-condensed present in the product. ${ }^{3} 32 \%$ of the S-units are condensed.

\begin{tabular}{|l|l|l|l|l|}
\hline Source & Conditions & $\mathrm{M}_{\mathrm{n}}(\mathrm{g} / \mathrm{mol})$ & $\mathrm{M}_{\mathrm{w}}(\mathrm{g} / \mathrm{mol})$ & D \\
\hline Walnut & A & 1096 & 1805 & 1.65 \\
\hline Walnut & B & 1174 & 2934 & 2.50 \\
\hline Walnut & $\mathrm{C}^{*}$ & 1248 & 2930 & 2.35 \\
\hline Pine & A & 1331 & 3071 & 2.31 \\
\hline Pine & B & 1319 & 3596 & 2.73 \\
\hline Beech & A & 1645 & 3743 & 2.28 \\
\hline Beech & B & 1368 & 4303 & 3.14 \\
\hline Cedar & A & 860 & 1626 & 1.89 \\
\hline Cedar & B & 1188 & 3292 & 2.77 \\
\hline
\end{tabular}

Table 2: Molecular weights of the obtained lignins. 


\begin{tabular}{|l|l|l|l|l|l|l|l|l|}
\hline Source & Conditions & Yield (\%) & S/G/H ratio & Total $\beta-O-4$ & S acetal (wt\%) & G acetal (wt\%) & H acetal (wt\%) & $\begin{array}{l}\text { Total acetal } \\
\text { yield (wt\%) }\end{array}$ \\
\hline Walnut & A & 5.0 & $45 / 46 / 9$ & 72 & 4.5 & 5.9 & 2.1 & 12.5 \\
\hline Walnut & B & 15.2 & $59 / 37 / 4$ & 74 & 3.6 & 4.7 & 1.0 & 9.3 \\
\hline Walnut & C & 16.2 & $65 / 33 / 2$ & 45 & 3.8 & 3.9 & 0.6 & 8.3 \\
\hline Pine & A & 3.5 & $0 />99 /<1$ & 59 & 0 & 9.9 & 0.3 & 10.2 \\
\hline Pine & B & 4.0 & $0 />99 /<1$ & 46 & 0 & 1.1 & 0 & 1.1 \\
\hline Beech & A & 5.4 & $63 / 37 / 0$ & 82 & 7.7 & 6.7 & 0 & 14.4 \\
\hline Beech & B & 13.9 & $83 / 17 / 0$ & 45 & 3.6 & 3.4 & 0 & 7.0 \\
\hline Cedar & A & 6.4 & $0 />99 /<1$ & 64 & 0 & 8.1 & 0.1 & 8.2 \\
\hline Cedar & B & 11.5 & $0 />99 /<1$ & 41 & 0 & 4.7 & 0 & 4.7 \\
\hline
\end{tabular}

Table 3: Acetal yields of lignin depolymerization. Yields of phenolic 2-arylmethyl-1,3-dioxolones (acetals) obtained from depolymerization of lignin from different sources. Conditions: $50 \mathrm{mg}$ lignin, $60 \mathrm{wt} \%$ ethylene glycol, $10 \mathrm{wt} \% \mathrm{Fe}(\mathrm{OTf})_{3}$, solvent: $1,4-$ dioxane, $140{ }^{\circ} \mathrm{C}(1 \mathrm{~mL}$ total volume), 15 minutes (step 7).

\begin{tabular}{|c|c|c|c|c|c|c|c|}
\hline Source & Conditions & \begin{tabular}{|l} 
Lignin extraction \\
yield (\%)
\end{tabular} & $\beta-O-4$ & $\beta^{\prime}-\mathrm{O}-4$ & Total $\beta-O-4$ & \begin{tabular}{|l|}
$\begin{array}{l}\text { Total acetal yield } \\
(\mathrm{w} t \%)\end{array}$ \\
\end{tabular} & $\begin{array}{l}\text { Overall acetal } \\
\text { yield corrected } \\
\text { for lignin } \\
\text { extraction yield } \\
(w t \%)^{1}\end{array}$ \\
\hline Walnut & $A$ & 5.0 & 34 & 38 & 72 & 12.5 & 0.63 \\
\hline Walnut & B & 15.2 & 20 & 54 & 74 & 9.3 & 1.41 \\
\hline Walnut & $C^{*}$ & 16.2 & 10 & 35 & 45 & 8.2 & 1.33 \\
\hline Pine & $A$ & 3.5 & 22 & 37 & 59 & 10.2 & 0.36 \\
\hline Pine & B & 4.0 & 7 & 39 & 46 & 1.1 & 0.04 \\
\hline Beech & A & 5.4 & 43 & 39 & 82 & \begin{tabular}{|l|l|}
14.4 \\
\end{tabular} & 0.78 \\
\hline Beech & B & 13.9 & 11 & 35 & 45 & 6.9 & 0.96 \\
\hline Cedar & $A$ & 6.4 & 28 & 36 & 64 & 8.2 & 0.52 \\
\hline Cedar & B & 11.5 & 7 & 34 & 41 & 4.7 & 0.54 \\
\hline
\end{tabular}

Table 4: Overall acetal yield corrected with extraction yield. Yields of phenolic 2-arylmethyl-1.3-dioxolanes (acetals) obtained from depolymerization of lignin from different sources corrected for lignin extraction yield. ${ }^{1}$ Calculation: $100^{*}($ lignin yield/100)*(total acetal yield/100). Conditions: 50 mg lignin, $60 \mathrm{wt} \%$ ethylene glycol, $10 \mathrm{wt} \% \mathrm{Fe}(\mathrm{OTf})_{3}$, solvent: 1,4-dioxane, $140{ }^{\circ} \mathrm{C}, 15$ min (reaction via step 6 \& work-up via step 7).

\section{Discussion}

The results from the extractions at different conditions and from different biomass sources reveal how the optimal conditions for lignin extraction with a relatively high content of $\beta-O-4$ linkages can vary depending on the source. For example, walnut extraction at harsher conditions (method B) provides a three-fold increase in the yield with a near retention of the amount of $\beta-O-4$ units, while for beech and cedar the yield increases but is accompanied by a significant drop in the amount of $\beta-\mathrm{O}-4$ units. On the other hand, for pine, the harsher extraction conditions provide very little benefit in yield and also lead to a lignin with a very low amount of $\beta-0-4$ units. This means that typically some form of optimization has to be involved to get the correct balance between the lignin yield and quality in the form of retention of the amount of $\beta-\mathrm{O}-4$ units in the obtained lignin material.

The large increase in the $\mathrm{M}_{\mathrm{w}}$ of the lignin material obtained from harsher extraction conditions demonstrates that under these conditions larger fragments can be extracted providing higher yields. However, at these conditions, additional fragmentation occurs, providing additional lower molecular weight material and thus increasing the polydispersity as can be clearly seen in the GPC graphs of walnut (Figure 5a) and cedar (Figure $5 \mathbf{d}$ ) in the form of the signal at $\sim 500 \mathrm{Da}$.

HSQC NMR is an important informative tool to provide comparative data on the quality of different lignins. It should be noted that in this procedure a standard HSQC experiment is performed, this is great for obtaining comparative data but is not necessarily quantitative due to differences in relaxation times. The high amount of linkages displayed for some lignins in Table 1 are overestimated. Quantitative HSQC experiments provide better results but cost significantly more NMR time, although alternatives exist ${ }^{29}$. In our experience, the numbers in Table 1 should be divided by a factor of a 1.3 to better reflect the actual amount of $\beta-0-4$ units per 100 aromatic units.

As previously mentioned, the reported results point out how finding optimum conditions can vary depending on the source in order to obtain the maximum monomer yields. For instance, when walnut is used as the starting material, the overall total acetals yield increases around two times if harsher conditions (method B) are employed for lignin extraction. However, this is mainly due to the great difference in lignin extraction yield, 
without affecting the $\beta-\mathrm{O}-4$ content. Differently, when pine is used milder extraction conditions (method A) are preferable. In fact, lignin extraction results in very similar yields in the two cases but harsher conditions cause a drop in $\beta-O-4$ units (especially non-etherified $\beta$-O-4 linkages) which can be the reason for such a low monomer yield, as indicated in the previous paragraph. A significant loss of non-etherified $\beta-O-4$ linkages can be observed as well in the cases of beech and cedar if conditions (method B) are applied for extraction which possibly leads to a lower monomers yield. However, the overall acetal yield does not differ that much depending on the extraction conditions. In fact, an approximate twofold increase in lignin extraction yield is observed for both biomass sources switching from methods A to B which compensates for the roughly two-fold decrease in monomer yield.

\section{Disclosures}

The authors report no conflict of interest

\section{Acknowledgements}

The work was funded by the European Union (Marie Curie ITN 'SuBiCat' PITN-GA-2013-607044, PJD, KB and JGdV), in addition to financial support from the European Research Council, ERC Starting Grant 2015 (CatASus) 638076 (AdS and KB) and the research programme Talent Scheme (Vidi) with project number 723.015.005 (KB), which is partly financed by the Netherlands Organisation for Scientific Research (NWO).

\section{References}

1. Tuck, C.O., Pérez E., Horváth, I.T., Sheldon, R.A., Poliakoff, M. Valorization of Biomass: Deriving More Value from Waste. Science. 337, 695-699 (2012).

2. Ragauskas, A.J. et al. Lignin Valorization: Improving Lignin Processing in the Biorefinery. Science. 344, 709-719 (2014).

3. Sun, Z., Fridrich, B., de Santi, A., Elangovan, S., Barta, K. Bright Side of Lignin Depolymerization: Toward New Platform Chemicals. Chemical Reviews, . 118 (2), 614-678 (2018).

4. Schutyser, W., Renders, T., Van den Bosch, S., Koelewijn, S.-F., Beckham, G.T., Sels, B.F., Chemicals from lignin: an interplay of lignocellulose fractionation, depolymerization, and upgrading. Chemical Society Reviews. 47 (3), $852-908$ (2018).

5. Kärkäs, M.D., Matsuura, B.S., Monos, T.M., Magallanes, G., Stephenson, C.R.J. Transition-metal catalyzed valorization of lignin: the key to a sustainable carbon-neutral future. Organic \& Biomolecular Chemistry . 14 (6), 1853-1914 (2016).

6. Deuss, P.J., Barta, K. From models to lignin: Transition metal catalysis for selective bond cleavage reactions. Coordination Chemistry Review. 306, 510-532 (2016).

7. Yokoyama, T. Revisiting the Mechanism of $\beta-\mathrm{O}-4$ Bond Cleavage During Acidolysis of Lignin. Part 6: A Review. Journal of Wood Chemistry and Technology. 35 (1), 27-42 (2014).

8. Sturgeon, M.R. et al. A Mechanistic Investigation of Acid-Catalyzed Cleavage of Aryl-Ether Linkages: Implications for Lignin Depolymerization in Acidic Environments. ACS Sustainable Chemistry and Engineering. 2 (3), 472-485 (2014).

9. Deuss, P.J., Scott, M., Tran, F., Westwood, N.J., de Vries, J.G., Barta, K. Aromatic monomers by in situ conversion of reactive intermediates in the acid-catalyzed depolymerization of lignin, Journal of the American Chemical Society . 137 (23), 7456-7467 (2015).

10. Jastrzebski, R., Constant, S., Lancefield, C.S., Westwood, N.J., Weckhuysen, B.M., Bruijnincx, P.C.A. Tandem Catalytic Depolymerization of Lignin by Water-Tolerant Lewis Acids and Rhodium Complexes, ChemSusChem. 9 (16), 2074-2079 (2016).

11. Kaiho, A., Kogo, M., Sakai, R., Saito, K., Watanabe, T. In situ trapping of enol intermediates with alcohol during acid-catalysed depolymerization of lignin in a nonpolar solvent. Green Chemistry. 17 (5), 2780-2783 (2015).

12. Huang, X., Zhu, J., Korányi, T.I., Boot, D.B., Hensen, E.J.M. Effective Release of Lignin Fragments from Lignocellulose by Lewis Acid Metal Triflates in the Lignin-First Approach. ChemSusChem. 9 (23), 3262-3267 (2016).

13. Kaiho, A., Mazzarella, D., Satake, M., Kogo, M., Sakai, R., Watanabe, T. Construction of di(trimethylolpropane) cross linkage and phenylnaphthalene structure coupled with selective $\beta-0-4$ bond cleavage for synthesizing lignin-based epoxy resins with controlled glass transition temperature. Green Chemistry . 18 (24), 6526-6535 (2016).

14. Lahive, C.W. et al. Advanced model compounds for understanding acid-catalyzed lignin depolymerization: Identification of renewable aromatics and a lignin-derived solvent. Journal of the American Chemical Society . 138 (28), 8900-8911 (2016).

15. Shuai, L., Saha, B. Towards high-yield lignin monomer production. Green Chemistry. 19 (16), 3752-3758 (2017).

16. Rinaldi, R. et al. Paving the Way for Lignin Valorisation: Recent Advances in Bioengineering, Biorefining and Catalysis. Angewandte Chemie International Edition. 55 (29), 8167-8215 (2016).

17. Schutyser, W., Renders, T., van den Bosch, S., Koelewijn, S.-F., Beckham, G.T., Sels, B.F. Chemicals from lignin: an interplay of lignocellulose fractionation, depolymerization, and upgrading. Chemical Society Reviews. 47 (3), $852-908$ (2018).

18. Constant, S. et al. New insights into the structure and composition of technical lignins: a comparative characterization study. Green Chemistry . 18 (9), 2651-2665 (2016).

19. Smit., A., Huijgen, W., Effective fractionation of lignocellulose in herbaceous biomass and hardwood using a mild acetone organosolv process. Green Chemistry. 19 (22), 5505-5514 (2017).

20. Lancefield, C.S., Panovic, I., Deuss, P.J., Barta, K., Westwood, N.J. Pre-treatment of lignocellulosic feedstocks using biorenewable alcohols: towards complete biomass valorization. Green Chemistry. 19 (1), 202-214 (2017).

21. Deuss, P.J., Lancefield, C.S., Narani, A., de Vries, J.G., Westwood, N.J., Barta, K. Phenolic acetals from lignins of varying compositions via iron (III) triflate catalysed depolymerization. Green Chemistry. 19 (12), 2774-2782 (2017).

22. Bauer, S., Sorek, H., Mitchell, V.D., Ibáñez, A.B., Wemmber, D.E., Characterization of Miscanthus giganteus Lignin Isolated by Ethanol Organosolv Process under Reflux Condition. Journal of Agricultural and Food Chemistry . 60 (33), 8203-8212 (2012).

23. Deuss, P.J. et al. Metal triflates for the production of aromatics from lignin. ChemSusChem. 9 (2), 2974-2981 (2016).

24. Nicholson, D. J., Leavit, A. T., Francis, R. C. A three-stage Klason method for more accurate determinations of hardwood lignin content. Cellulose Chemistry and Technology . 48 (1-2), 53-59 (2014). 
25. Sun, Z. et al.Complete lignocellulose conversion with integrated catalyst recycling yielding valuable aromatics and fuels. Nature catalysis . $\mathbf{1}$, $82-92$ (2018).

26. Lancefield, C.S., Ojo, O.S., Tran, F., Westwood, N.J. Isolation of Functionalized Phenolic Monomers through Selective Oxidation and C-O Bond Cleavage of the $\beta-O-4$ Linkages in Lignin. Angewandte Chemie International Edition. 54 (1), 258-262 (2015).

27. Rahimi, A., Ulbrich, A., Coon, J.J., Stahl, S.S. Formic-acid-induced depolymerization of oxidized lignin to aromatics. Nature. 515, 249-252 (2014).

28. Bosque, I., Magallanes, G., Rigoulet, M., Kärkäs, M.D., Stephenson, C.R.J. Redox Catalysis Facilitates Lignin Depolymerization, ACS Central Science. 3 (6), 621-628 (2017).

29. Sette, M., Wechselberger, R., Crestini, C., Elucidation of Lignin Structure by Quantitative 2D NMR. Chemistry - A European Journal. 17 (34), 9529-9535 (2011). 